\title{
LIMITED LIABILITY, MORAL HAZARD, AND RISK TAKING: A SAFETY NET GAME EXPERIMENT
}

\author{
SASCHA FÜLLBRUNN and TIBOR NEUGEBAUER*
}

\begin{abstract}
We model the safety net problem as a social dilemma game involving moral hazard, risk taking, and limited liability. The safety net game is compared to both an individual decision task involving full liability and the deterministic public goods game. We report experimental data to show that limited liability leads to higher risk taking in comparison to full liability; nevertheless, the difference is much smaller than predicted by theory. In the safety net game, subjects behave as if socially responsible for the losses they impose on the group. With repetition, nevertheless, a gradual emergence of the moral hazard problem arises. (JEL C9, D7, D8, H4, I1, I3)
\end{abstract}

\section{INTRODUCTION}

In many parts of the industrialized world it is believed that the more equally and widely we share the risks of illness, unemployment, and poverty, the better off is the population as a whole. A variety of social safety nets and statesponsored insurance schemes are the result of the policies which prevent any individual from falling into misery beyond a certain level. In its core, a social safety net is a transfer of risk and liability from the citizen to society. Detractors of safety nets regard these risk and liability transfers not as the solution to misfortune, but as the problem. It is argued that the assumption of individual liabilities by the society reduces the incentives to mitigate risks and adversely affects people's behavior. This moral hazard problem (Pauly 1968) was mentioned in the context of recent governmental bailouts. The argument that

*We wish to thank two anonymous referees and the editor of the journal for helpful comments, Abdolkarim Sadrieh and the Faculty of Economics and Management of University of Magdeburg for the use of their MaXLab, and Marina Schröder and Harald Wypior for professional lab assistance. S.F. has been supported by the National Research Fund, Luxembourg (PDR 09 044), and the UL internal project F2R-LSF-PUL-10IDIA. We acknowledge funding of the experiments through the internal UL project F2R-LSFPUL-09BCCM.

Füllbrunn: Research Fellow, Max Planck Institute of Economics, Strategic Interaction Group, Kahlaische Straße 10, D-07745 Jena, Germany. Phone +49 3641686646 , Fax +493641 686 667, E-mail fuellbrunn@econ.mpg.de

Neugebauer: Professor of Finance, Université de Luxembourg, Faculté de Droit, d'Economie et de Finance, Luxembourg School of Finance, 4, rue Albert Borschette, L-1246 Luxembourg. Phone +352 466644 6285, Fax +352466644 6835, E-mail Tibor.Neugebauer@uni.lu gains are privatized, but losses are socialized suggests a susceptibility to excessive risk taking in safety nets.

Beyond its theoretical importance, very few empirical studies have provided evidence for the significance of moral hazard, and these studies have produced mixed results. Some authors indicate higher risk taking among insured institutions or subjects (Dionne et al. 2005; Grossman 1992), whereas others find no difference in the comparison to the uninsured (Abbring, Chiappori, and Pinquet 2003). Surprisingly little evidence has been reported on the abuse of safety nets in health care (e.g., Breyer, Zweifel, and Kifmann 2004; Wolfe and Goddeeris 1991), or unemployment (e.g., Pallage and Zimmermann 2001).

Generally, the collection of reliable empirical data on higher risk taking rates under limited liabilities vis-à-vis full liability is a challenging task. Risk taking behavior can only be estimated indirectly from empirical data, and specification problems (Abbring, Chiappori, and Pinquet 2003) loom frequently, for instance in the case of private insurance, moral hazard and adverse selection are hard to tell apart. So, it is difficult to judge the significance of moral hazard in safety nets based on empirical data alone. As an alternative and additional data source

\section{ABBREVIATIONS}

IND: Individual Decision Problem

PGG: Public Goods Game

SNG: Safety Net Game 
we propose economics experiments. Thus, the impact of liability changes on risk taking can be studied through direct observation of human behavior, and problems of data ambiguity can be excluded by design.

We model the safety net problem as a social dilemma game in which players are exposed to the risk of individual losses that are assured by the group. The behavior in this game where individual players face limited liability is benchmarked with the behavior under full liability, where individual decision making occurs in the absence of a safety net. Given standard assumptions, the equilibrium predictions for both scenarios are divergent. For the full-liability case, expected utility maximization requires a maximum level of loss avoidance measures. In contrast, minimum loss avoidance levels are exerted in the unique equilibrium of the safety net game. Our experimental data show both predictions are too extreme. In both treatments, the average loss avoidance levels are chosen in the interior upper half of the action space. The evidence thus suggests that subjects do not free-ride on the safety net as much as the theoretical solution suggests. Similar evidence has been reported in the experimental literature on public goods games following the seminal paper by Isaac, Walker, and Thomas (1984), which share similarities to our safety net game. In particular our study closely relates to public goods experiments involving risk. This literature, which we briefly survey in the following section, investigates how elements of risk affect behavior in public goods games. We contribute to this literature as we use a linear public goods game in a loss framing as a second benchmark treatment to the safety net game. Both games yield the same equilibrium predictions, but we report steadily higher loss avoidance levels for the safety net game. In comparison to this benchmark treatment, complete free riding is largely reduced and loss avoidance levels are shifted to the upper half of the action space. We argue that subjects display a higher level of social responsibility in the safety net game than in the public goods game, because they react to the occurrence of a loss in an adaptive way. Other things equal, they increase rather than decrease their avoidance levels after a loss has occurred.

The article is organized as follows. After a short review of the experimental literature in Section II we consider the theoretical framework of the safety net game and its benchmarks in Section III. In Section IV we introduce the experimental parameters and detail the procedures of the experimental design. In Section $\mathrm{V}$ we report the experimental results, and in Section VI we conclude.

\section{REVIEW OF THE EXPERIMENTAL LITERATURE}

The main stream of experimental research on problems of moral hazard focuses on the two-player principal-agent problem (Holmstrom 1979). Testing the subgame perfect equilibrium of the game including the incentive compatibility requirement (Berg et al. 1992; Epstein 1992; Güth et al. 1998; Keser and Willinger $2000,2007)$ and reciprocity in work contracts (Anderhub, Königstein, and Gächter 2002; Fehr, Gächter, and Kirchsteiger 1997; Gächter and Königstein 2009) have been the focal topics. The results of this research suggest that if the agent is promised a fair reward by the principal, the agent will reciprocate this fairness. The moral hazard problem turns into a threat of negative reciprocity for perceived unfairness. Such reciprocity and fairness concerns are an issue in our safety net game, too. We report that the individual loss avoidance levels are correlated to the lagged losses in the group. Nonetheless, in comparison to the principal-agent literature the safety net game involves multiple players who make their decisions simultaneously.

Closely related to our study are public goods experiments that investigate the effects of risk on cooperation. Generally, the results of this literature vary depending on the experimental design. Two categories of risky public goods games have been studied. The first one which includes the safety net game involves the pooling of individual risks, and the second category involves the pooling of individual contributions to a risky public good. The existing experimental investigations in the first category have found mixed results (Berger and Hershey 1994; Gong, Baron, and Kuhnreuter 2009). Berger and Hershey report smaller and Gong, Baron, and Kuhnreuter report larger cooperation levels when individual risks are involved. Both studies consider binary choice situations, where the risk of a loss can either be fully accepted or the loss can be fully insured. In contrast to these studies, our safety net game has a rather continuous action space, where the amount of risk is subject to choice. We observe that most chosen loss avoidance levels are in the interior upper half of the action space, and cooperation levels are larger than in the deterministic public 
goods game. The action space of the referenced studies thus does not contain the chosen actions of our experiment. The studies of Gangadharan and Nemes (2009), Dickinson (1998), Hasson, Löfgren, and Visser (2010), and Burger and Kolstad (2009) fall into the second category of risky public goods games where the sum of individual contribution to the public good is subject to risk of being lost. ${ }^{1}$ A chance event which follows up on subjects' contributions to the public good determines with fixed probability if subjects receive a payoff from their contributions to the public good or if the contributions are lost. Gangadharan and Nemes (2009) and Dickinson (1998), furthermore, consider a treatment where, similarly to our safety net game but applied to the group contribution, the probability of loss avoidance is positively correlated with the sum of contributions. In comparison to our safety net game environment, loss avoidance depends not only on the individual contribution, but also on the contribution of the others. Therefore it may not be surprising that contribution levels are lower in their risky treatment than in their baseline treatment where such risk does not exist.

The literature abounds on studies related to our public goods control treatment; see surveys by Hey (1991) or Ledyard (1995). Differing from the experimental main stream approach, however, we apply a loss framing in the sense of Kahneman and Tversky (1981). Shanley and Grossman (2007) conducted public goods experiments in a negative framing and in a positive framing to observe higher cooperation levels in the standard positive framing. We have no control to investigate if our loss framing leads to similar relative results.

Finally, Di Mauro (2002) studies a related experiment to our full liability treatment, where subjects make an individual decision under risk or uncertainty by choosing a loss avoidance level to decrease the probability of the loss. She compares the effect of risk and uncertainty, and reports higher average loss avoidance levels under uncertainty than under risk.

\section{THEORETICAL CONSIDERATIONS}

This section introduces and derives the theoretical predictions of the safety net game. The safety net game involves collective risk sharing in the group. Individuals take costly

1. Only the experimental literature is surveyed in this section. A related theoretical study has recently been published by Lohse, Robledo, and Schmidt (2012). loss avoidance measures, but collectively indemnify the incurred losses. Thus the originator of a social loss has limited liability as he or she shares the loss in the same way as everyone else. The alternative to this scenario is full liability, where, in absence of a safety net, individuals must cover their entire loss alone.

\section{A. The Individual Decision Problem (IND):Full Liability}

To avoid a loss $L>0$, an individual $i$ chooses a loss avoidance level $x_{i} \in[0 ; \bar{x}]$. For the given loss avoidance level $x, p(x)$ is the conditional probability of loss avoidance of a Bernoulli distribution. The derivative of the probability function with respect to $x$, denoted by $p^{\prime}(x)$, is strictly positive for all intermediate loss avoidance levels. At the boundaries, it is assumed the loss is incurred (avoided) with certainty if loss avoidance is 0 (at the maximum level). The unit cost of loss avoidance is $c>0$. The individual maximizes the von Neumann-Morgenstern utility function described in Equation (1), where $a>c \bar{x}+L$ denotes the amount of owned assets, and $u_{i}(y)$ is the utility of consequence $y$.

(1)

$$
\begin{aligned}
U_{i}\left(x_{i} ; L\right)= & p\left(x_{i}\right) u_{i}\left(a-c x_{i}\right) \\
& +\left(1-p\left(x_{i}\right)\right) u_{i}\left(a-c x_{i}-L\right) .
\end{aligned}
$$

We assume the loss is significant, that the individual is risk averse or risk neutral and that he or she seeks to maximize expected utility. For our purpose of predicting the individual decisions in the experiment, a case of special interest is the extreme one, where utility maximization implies the choice of the maximum loss avoidance level. A sufficient assumption for such a corner solution is given by $p^{\prime}\left(x_{i}\right) L \geq c \forall x_{i} \in(0, \bar{x})$, that is, the marginal advantage of loss avoidance weakly exceeds its marginal costs, and at the upper boundary $L \geq c \bar{x}, x_{i}=\bar{x}$. This assumption involves the economically interesting cases for the full liability case. Under standard rationality assumptions, thus, only risk seekers choose a non-maximal loss avoidance level.

\section{B. The Safety Net Game (SNG): Limited Liability}

Consider a group of $n$ individuals who face the described IND, but who decide to collectively and equally share all individual losses incurred within the group. The thus created safety net implies that each player has the same 
liability for his or her own loss as for the losses of everyone else. The expected utility thus depends also on the loss avoidance levels taken by the other individuals in the group. In the SNG the individual maximizes the probability weighted utility of each possible outcome. goods game by Isaac, Walker, and Thomas (1984). In contrast to the standard gains framing, however, our PGG involves a loss framing. ${ }^{3}$

Consider a group of $n$ individuals who simultaneously decide on the reduction of an individual loss to be borne by the group. By

$$
\begin{aligned}
U_{i}\left(x_{i} ; x_{-i}, L\right)= & \prod_{k=1}^{n} p\left(x_{k}\right) \times u_{i}\left(a-c x_{i}\right) \\
+ & {\left[\sum_{j_{1}=1}^{n}\left(1-p\left(x_{j 1}\right)\right) \times \prod_{k \neq j_{1}} p\left(x_{k}\right)\right] \times u_{i}\left(a-c x_{i}-L / n\right) } \\
+ & {\left[\sum_{j_{1}=1}^{n} \sum_{j_{2} \neq j_{1}}\left(1-p\left(x_{j_{1}}\right)\right) \times\left(1-p\left(x_{j_{2}}\right)\right) \times \prod_{k \neq\left\{j_{1}, j_{2}\right\}} p\left(x_{k}\right)\right] \times u_{i}\left(a-c x_{i}-2 L / n\right) } \\
& \ldots \ldots \sum_{j_{n-1} \neq\left\{j_{1}, j_{2}, \ldots, j_{n-2}\right\}}\left(1-p\left(x_{j_{1}}\right)\right) \times \cdots \times\left(1-p\left(x_{j_{n-1}}\right)\right) \\
+ & {\left[\sum_{j_{1}=1}^{n} \ldots \ldots(n)\right.} \\
& \left.\times p\left(x_{k \neq\left\{j_{1}, \ldots, j_{n-1}\right\}}\right)\right] \times u_{i}\left(a-c x_{i}-(n-1) L / n\right) \\
+ & \prod_{j=1}^{n}\left(1-p\left(x_{j}\right)\right) \times u_{i}\left(a-c x_{i}-L\right) .
\end{aligned}
$$

Taking the loss avoidance levels of the others as given, a necessary condition for positive equilibrium levels of loss avoidance is $p^{\prime}\left(x_{i}\right) L / n \geq$ $c \forall x_{i} \in(0, \bar{x})$ or at the boundary $L / n \geq c \bar{x}, x_{i}=$ $\bar{x}$. In contrast, if the marginal costs exceed the individual marginal benefit of loss avoidance, $p^{\prime}\left(x_{i}\right) L / n<c \forall x_{i},{ }^{2}$ as we are going to assume in the experiment, expected utility maximization implies that every individual in the SNG chooses a zero loss avoidance level. Maximization of the expected collective welfare, however, would lead to avoidance of any losses, owed to the above made assumption $p^{\prime}\left(x_{i}\right) L \geq c \forall x_{i}$. By the standard backward induction argument, the equilibrium of the finitely repeated game involves zero loss avoidance levels in each stage.

\section{The Linear Public Goods Game (PGG): The Deterministic Benchmark}

As deterministic control to the safety net game we consider the well-known linear public

2. As cost, loss, and loss avoidance levels are nonnegative numbers, the lower bound is sufficiently defined. choosing the loss avoidance level $x_{i} \in[0 ; \bar{x}]$ the individual reduces the loss to the group at a constant unit cost $c$. The group members equally share the sum of non-avoided individual losses. Assume individuals symmetrically maximize the following utility function.

$$
\begin{aligned}
& U_{i}\left(x_{i} ; L\right) \\
& \quad=u_{i}\left(a-c x_{i}-\sum_{j=1}^{n}\left(L-b x_{j}\right) / n\right) ; x_{j} \in[0 ; \bar{x}] .
\end{aligned}
$$

Rearranging terms, $a-L$ is equivalent to the endowment of the individual in the standard gain

3. An equivalent parameterization has been used in the standard gain framing design of the linear public goods game, for instance, by Croson, Fatas, and Neugebauer (2005). In an early draft of this study, we used a gain framing for the treatments IND and SNG. The results were qualitatively equal. However, since there were some subject pool differences, we do not report the results here. Nonetheless the data and the early draft can be obtained upon request. 
framing of the linear PGG, and the marginal per capita return of a unit contributed to loss avoidance is $b / n$. Thus, our setting is equivalent to Isaac, Walker, and Thomas (1984). For given $b \in(1, n)$, as standard assumption, complete loss avoidance is collectively rational. However, given the contribution of the others, individuals' best response is to contribute nothing in group interest. In accordance with the SNG, collective free-riding occurs in the unique equilibrium of the finitely repeated game. Therefore, both the PGG and the SNG are social dilemmas. In contrast to the SNG where the individual contributes to a reduction of the loss probability, however, the individual contributes to a proportional reduction of the loss in the PGG.

\section{EXPERIMENTAL DESIGN AND TESTABLE HYPOTHESIS}

The experimental design includes three treatments; the SNG as described in Section III.B, the IND in which the individual faces full liability of the incurred risk as described in Section III.A, and the deterministic PGG control treatment as described in Section III.C.

\section{A. Parameterization}

The following experimental parameter values were chosen. Subjects faced a (gross) endowment of $a=150$, marginal group return $b=2$, loss avoidance costs $c=1$, and a potential loss of $L=100$. Subjects were asked to choose a loss avoidance level between zero and the maximum level $\bar{x}=50$. All amounts refer to Eurocent. In the SNG and IND treatments, individual threshold levels $s_{i}$ were drawn from a uniform distribution on the interval $\{1, \ldots, 50\}$ to determine if the individual loss was incurred or avoided. If the chosen loss avoidance level weakly exceeded the threshold level, that is $x_{i} \geq$ $s_{i}$, the loss was avoided; otherwise, that is, if the subject's loss avoidance level was lower than the threshold level, the loss was incurred. As pointed out above, the subject incurred (avoided) the loss with certainty by choosing the zero (maximum) loss avoidance level. The SNG and the PGG treatments involved groups of size $n=4$ in a partners setting. Hence, our parameterization implies the opposing corner solutions for the IND treatment, $p^{\prime}\left(x_{i}\right) L=2 \geq 1=c \forall x_{i}$, and the SNG treatment, $p^{\prime}\left(x_{i}\right) L / n=25 / 50<$ $1=c \forall x_{i}$.

Table 1 records the resulting payoff functions along with the theoretical equilibrium prediction on loss avoidance levels and payoffs. The event of loss avoidance is indicated by the binary variable $I_{i}$, which takes value 1 if $s_{i}>x_{i}$ and 0 otherwise. Therefore, treatments are similar in that each unit increase in $x_{i}$ reduces the expected loss incurred through subject $i$ by two units. However, treatments are different in the allocation of losses. While in IND each additional Eurocent spent on loss avoidance increases the expected per capita return by two, in SNG and PGG, each additional Eurocent spent on loss avoidance increases the expected per capita return by one half because losses are allocated in equal shares among group members. Although the expected payoffs in SNG and PGG are equivalent for any given strategy profile, the increase of the expected payoff in SNG is affected through a reduction in the loss probability, whereas in PGG it is affected through an increased cash payoff.

\section{B. Procedures}

Each treatment involved ten periods (i.e., repetitions) with feedback information. In the SNG treatment, subjects received private information on their own chosen loss avoidance levels, their individual threshold levels, their own losses, the sum of group losses including the own loss, and their individual payoff. This information was

TABLE 1

Experimental Setting and Prediction

\begin{tabular}{|c|c|c|c|}
\hline Treatment & Payoff Function & $\begin{array}{l}\text { Predicted Loss } \\
\text { Avoidance Level }\end{array}$ & $\begin{array}{l}\text { Predicted } \\
\text { Payoff }\end{array}$ \\
\hline IND & $150-x_{i}-100 I_{i}, I_{i}=\left\{\begin{array}{l}0, \text { if } x_{i} \geq s_{i}, s_{i} \sim U\{1,2, \ldots, 50\} \\
1, \text { otherwise }\end{array}\right.$ & 50 & 100 \\
\hline SNG & $150-x_{i}-\sum_{j=1}^{4} \frac{100}{4} I_{j}, I_{j}=\left\{\begin{array}{l}0, \text { if } x_{j} \geq s_{j}, s_{j} \sim U\{1,2, \ldots, 50\} \\
1, \text { otherwise }\end{array}\right.$ & 0 & 50 \\
\hline PGG & $150-x_{i}-\sum_{j=1}^{4} \frac{100-2 x_{j}}{4}$ & 0 & 50 \\
\hline
\end{tabular}


permanently available in a table for each past period. In the IND and the PGG treatments, the received information was equivalent to the one of the SNG treatment. However, no information about the other subjects' losses was available in the IND treatment, and no threshold levels were recorded in the PGG treatment.

The information on the sum of group losses is less transparent in the SNG than in the PGG. In the PGG treatment, the losses fully reveal the sum of loss avoidance levels while, in the SNG treatment, losses only reveal that subjects have exerted loss avoidance levels different from the maximum. If a loss occurs, however, one cannot tell if it is owed to low loss avoidance levels or rather owed to bad luck. This lack of transparency provokes the moral hazard or hidden action problem highlighted in the literature. In our setting this problem has two dimensions. On one hand, free-riders have an advantage as they are able to cover their low cooperation levels without fearing immediate punishment; on the other hand, cooperators have a disadvantage as they may have to incur higher loss avoidance levels to show their cooperation. If the effect of the free-rider advantage is larger (smaller) than the effect of cooperators' disadvantage, loss avoidance levels should be lower (higher). The moral hazard literature proposes the dominance of the free-rider advantage only, and neglects the effect on cooperators.

Subjects participated only in one session and interacted with exactly three other subjects. Each subject participated in the SNG treatment and in one other treatment, depending on the session either in IND or PGG. Treatments were conducted in different orders, thus we control for an order effect. Half the subjects played the SNG treatment in the first run and the other half played the SNG treatment in the second run of the session. In total, we conducted four sessions to be identified by the following treatment orders: IND-SNG, SNG-IND, PGG-SNG, and SNG-PGG. The comparison of the two first sessions informs us about an order effect with respect to risk taking when being switched from limited to full liability, and the comparison of the latter two sessions informs us about a behavioral change when being switched from the nonrisky to the risky PGG and vice versa. Note again, groups remained the same in the first and the second treatment of a session and were aware of this fact in the second run. In the first run, however, subjects were uninformed about the second treatment.
We conducted the sessions at the Magdeburg Experimental Laboratory (MaXLab). Subjects were undergraduate students of the Ottovon-Guericke Universität Magdeburg, Germany. The experimental software was programmed using z-Tree (Fischbacher 2007); participants were recruited via ORSEE (Greiner 2004). At the beginning of each treatment, the instructions were read aloud. Prior to interacting, subjects ran through a stand-alone computerized comprehension test. ${ }^{4}$ At the end of the session, we debriefed subjects via an onscreen questionnaire. $^{5}$ Given that all subjects passed the test and given their replies in the debriefings, we are confident that the instructions were understood.

\section{EXPERIMENTAL RESULTS}

We conducted four sessions with 28 subjects each. Each of the 112 subjects participated in exactly one session including two experimental treatments of ten periods. Per session, we collected seven independent observations of foursubject groups. Since every subject participated in the SNG treatment and one other treatment, the data consist of 28 independent observations for the SNG treatment, and 14 independent observations each for the PGG and IND treatments. The experimental design involves both a within-subjects component (as each subject participated in two different treatments) and a between-subjects component (as each subject participated in one session of the SNG). Each experimental session lasted about an hour. Subjects' average earnings were $17.44 €$ in the sessions that included the IND treatment and $14.83 €$ in the sessions that included the PGG treatment. No additional show-up fee was paid. On the basis of the following result, subsequent analysis is conducted on the pooled data regardless of the treatment order.

4. The translated instructions and a description of the onscreen-test are appended to the article.

5. As the experiment involves decision making under risk, we asked subjects to state their risk preferences on a scale from 1 to 7 . This task was not incentivized. The average reported level was 4.375 , indicating risk aversion rather than risk loving. Correlating 28 individual average loss avoidance levels in IND and PGG and 56 in SNG to the stated level of risk aversion the Spearman rank correlation coefficients are .347 in IND (the $p$ value of the one-sided test is .035), .204 in PGG, and -.029 in SNG. For IND, thus, we find unincentivized evidence for a positive relationship between the stated levels of risk aversion and the chosen loss avoidance levels. We find no comparable evidence for the other treatments. 
TABLE 2

Order Effect: Random Effects Regression of Average Loss Avoidance Levels

\begin{tabular}{lccc}
\hline Independent Variables & (1) SNG & (2) PGG & (3) IND \\
\hline Run2Dummy & $5.813(3.793)$ & $-0.452(7.065)$ & $1.243(2.155)$ \\
Run2Dummy $\times$ Period & $-0.249(0.245)$ & $-0.009(0.387)$ & $0.165(0.167)$ \\
Period & $-1.334^{* * *}(0.173)$ & $-1.164^{* * *}(0.274)$ & $0.216^{*}(0.118)$ \\
Constant & $33.43^{* * *}(2.682)$ & $27.60^{* * *}(4.996)$ & $41.82^{* * *}(1.524)$ \\
Observations & 280 & 140 & 140 \\
Independent groups & 28 & 36.49 & 14 \\
Wald test & 144.1 & 15.06 \\
\hline
\end{tabular}

Notes: The dependent variable is the average loss avoidance level of a group in period $t$. Run2Dummy takes value 0 for the first and 1 for the second ten periods. Period takes values $\{1, \ldots, 10\}$ according to the period of the treatment. Standard errors in parentheses.

${ }^{* * *} p<.01,{ }^{* *} p<.05,{ }^{*} p<.1$

Observation 1: We observe no order effect. There are no significant differences between the firstrun and second-run loss avoidance levels for any treatment (SNG, PGG, and IND).

The observation is supported by the outcomes of the random effects dummy regression of group loss avoidance levels on a time trend. ${ }^{6}$ The results are recorded in Table 2 for each treatment; and the results are detailed for the SNG treatment in Appendix Table A1. The binary variable Run2Dummy takes value 0 for the first run and 1 for the second run of a session. This variable is interacted on the intercept and slope $($ Run 2 Dummy $\times$ Period $)$. Thus we test for order effects on initial levels and dynamics. The data show that both of these coefficients are not significant at the $10 \%$ level. In favor of higher statistical power, hence, we subsequently focus on the pooled data per treatment. ${ }^{7}$

Observation 2: Loss avoidance levels decline in the SNG and the PGG treatments.

The variable Period in Table 2 reveals the time trend of the treatments. The coefficient is significantly negative for the SNG and the PGG. The positive coefficient for the IND is only marginally significant. The effects are illustrated for every treatment in Figure 1 which displays the average loss avoidance levels and plots the differences between treatments. Over all periods, the average loss avoidance levels

6. Our panel data regression results are in line with the outcomes of the Hausman test; that is, unless the coefficients of the fixed effects model and the random effects model are significantly different, the random effects regression results are reported.

7. Nevertheless, the subsequently reported observations are fully in line with each treatment order. were $57 \%, 42 \%$, and $88 \%$ of the endowment in the SNG, PGG, and IND treatments (see also Table A2).

Observation 3: Loss avoidance levels in the SNG treatment are lower than in the IND treatment and higher than in the PGG treatment.

Statistical support for Observation 3 is given by the random effects dummy regression of group loss avoidance levels on a time trend across treatments, which is recorded in Table 3. The regression involves the binary treatment variable $S N G$ which takes value 1 for treatment SNG and 0 otherwise. In column 5 of Table 3, the significant positive effect of this treatment variable indicates the absolutely higher loss avoidance levels in the SNG compared to the PGG. Obviously, the risk exposure in the SNG influences behavior. The treatment variable is also interacted on the slope $(S N G \times$ Period $)$. The coefficient, however, indicates no difference in the decline of loss avoidance levels. Figure 1 shows that the loss avoidance levels of these two treatments decline in parallel. Relative to the IND treatment, on the other hand, we observe a negative treatment effect of the SNG in both average loss avoidance level and dynamics as indicated in column 6 . The loss avoidance levels of the IND are both absolutely higher and non-decreasing over periods.

Having made these observations, the questions arise in what way and why subjects in the SNG exert higher loss avoidance levels than in the PGG? In reply to these questions we start by examining the observed behavior in the distributions of loss avoidance levels before we turn to the between-treatments analysis of the dynamics. 
FIGURE 1

Average Loss Avoidance Levels

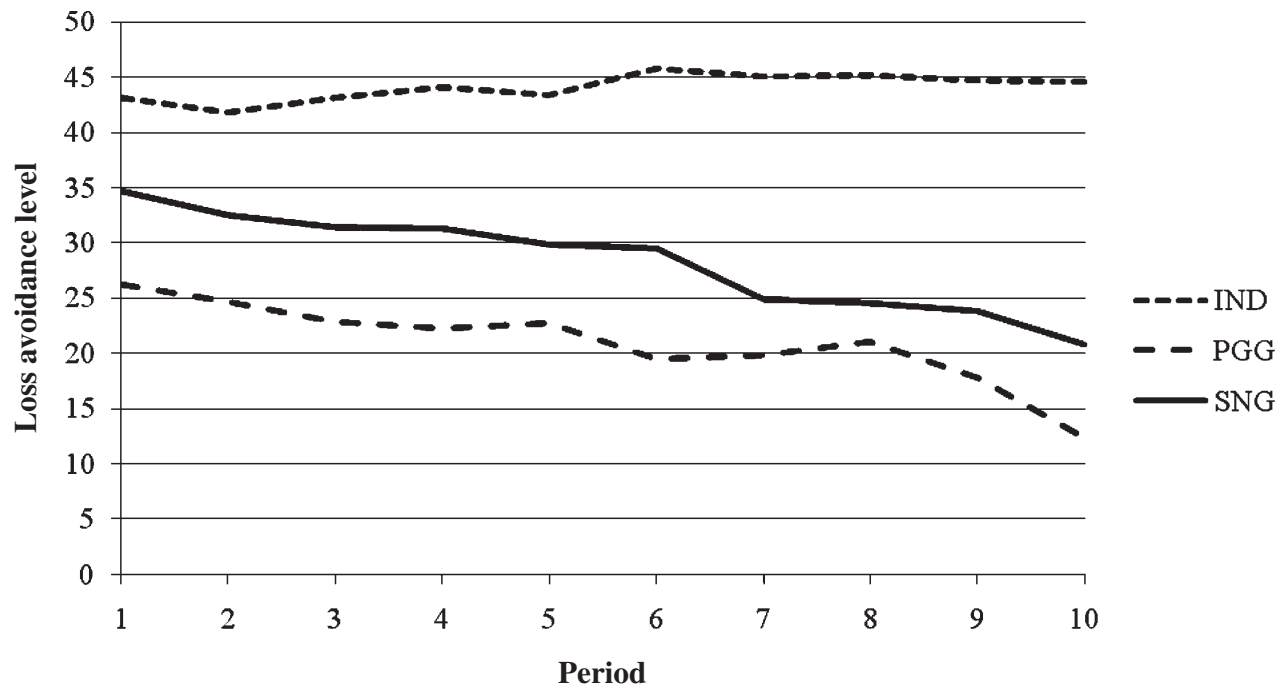

TABLE 3

Treatment Effect: Random Effects Regression of Average Loss Avoidance Levels

\begin{tabular}{lcc}
\hline $\begin{array}{l}\text { Independent } \\
\text { Variables }\end{array}$ & (5) PGG & (6) IND \\
\hline$S N G$ & $7.296^{* * *}(1.574)$ & $-4.451^{* * *}(1.467)$ \\
$S N G \times$ Period & $-0.290(0.245)$ & $-1.757^{* * *}(0.229)$ \\
Period & $-1.168^{* * *}(0.200)$ & $0.299(0.187)$ \\
Constant & $29.04^{* * *}(2.192)$ & $40.79^{* * *}(1.927)$ \\
Observations & 420 & 420 \\
Independent groups & 28 & 28 \\
Wald test & 189.5 & 475.7 \\
\hline
\end{tabular}

Notes: The average loss avoidance level of a group in period $t$ is the dependent variable in PGG and IND sessions. $S N G$ takes value 1 for the SNG and 0 for the other treatments. Period takes values $\{1, \ldots, 10\}$ according to the period of the treatment. Standard errors in parentheses.

${ }^{* * *} p<.01,{ }^{* *} p<.05,{ }^{*} p<.1$.

The distributions are plotted in Figure 2 for each treatment. The distribution of the SNG generally dominates the one of the PGG; this pattern is persistent from the first to the last period of the experiment. ${ }^{8}$ The distributions are presented along with a two-step OLS regression

8. The two-tailed Kolmogorov-Smirnov betweensample test confirms the dominance of the aggregate distribution between samples for the first ten periods $(p=.000)$. The Figures A1-A3 in the Appendix show the evolution of distributions over the periods for the three treatments. which smooth the distributions on the segments $[0, \ldots, 24]$ and $[25, \ldots, 49]$, respectively. ${ }^{9}$ Compared to the distribution of the PGG which is rather kinkless over the two segments, as also indicated by the regression lines, the distribution of the SNG exhibits an important jump at loss avoidance Level 25. Less mass of the distribution of loss avoidance levels is located toward the lower half of the endowment in the SNG than in the PGG treatment.

\footnotetext{
Observation 4: There are fewer observations of low loss avoidance levels [0,.., 24] and more of intermediate loss avoidance levels [25, .., 49] for the SNG treatment than for the PGG treatment. Compared to the IND, there are fewer maximum loss avoidance levels and more low loss avoidance levels in the SNG.
}

Statistical support for Observation 4 is given by the two-tailed Wilcoxon signed ranks test for matched samples. The null-hypothesis of equal loss avoidance levels in the SNG and the PGG treatments is rejected at the $5 \%$ level for both

9. The slope and intercept of the stepwise linear regressions are recorded in Table A3 of the Appendix, where also the linear OLS regression is reported for the purpose of comparison. The squared deviation of the loss avoidance level distribution from the regression-slope without step is only 1.5 times the sum of squared deviations of the two-step regression in case of the PGG, whereas 11.47 times and 8.93 times for the SNG and IND, respectively. The reported determination coefficients show the effect, too. 
FIGURE 2

Distribution of Loss Avoidance Levels and Stepwise Linear Approximation

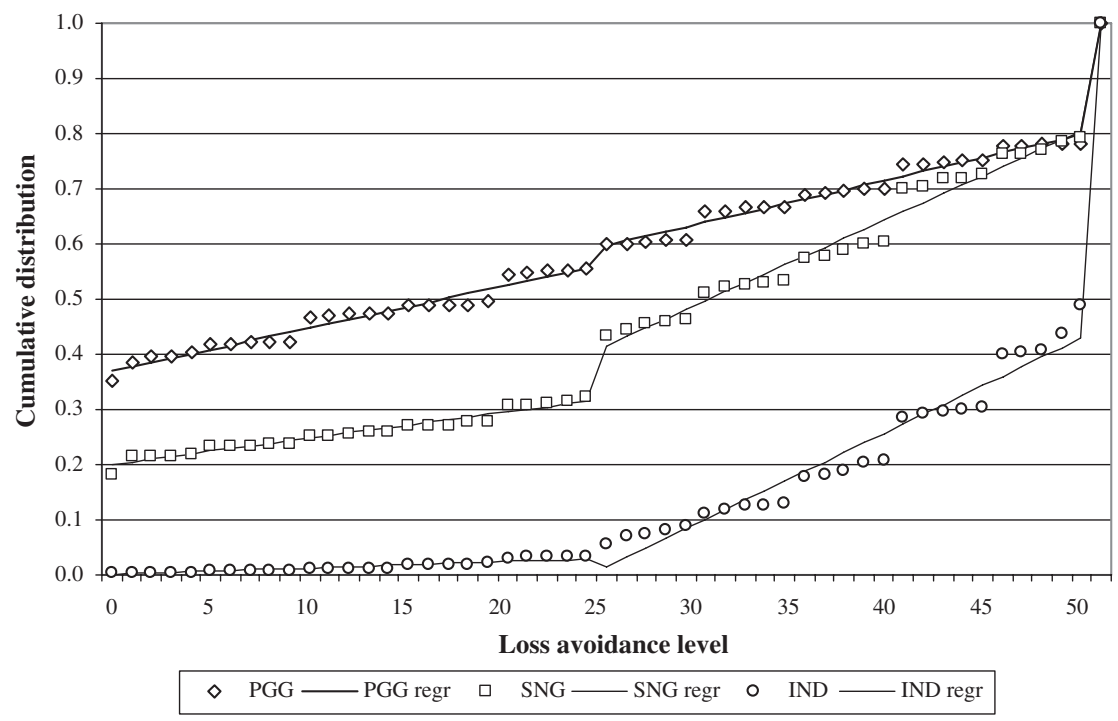

low loss avoidance levels $(p=.005),{ }^{10}$ and for intermediate loss avoidance levels $(p=.003)$. The frequencies of high loss avoidance levels, $x_{i}=\bar{x}$, are not significantly different $(p=$ .722). In line with the equilibrium predictions, the equivalent one-tailed Wilcoxon signed ranks test across the IND and the SNG indicates significant differences for the low loss avoidance levels $(p=.001)$ and for the high loss avoidance levels $(p=.001)$. However, the two-tailed Wilcoxon signed ranks test does not indicate significant differences for the intermediate levels $(p=.306)$.

Observation 4 suggests a shift between the distributions of the PGG and the SNG from the low loss avoidance level, most prominently zero loss avoidance levels, to the intermediate level. ${ }^{11}$ Compared to the IND, the support of

10. The between-treatments test indicates in the same direction, although the Mann-Whitney test is not significant $(p=.149)$.

11. We observe that the zero loss avoidance level is exerted in $18 \%$ of the decisions in the SNG. In the first and last period the relative frequencies are 10\% and $27 \%$ (see Figure A1 and Table A2). These numbers are not only much smaller than the theoretically predicted $100 \%$, but also half the size and significantly different to the PGG treatment where the level is $35.36 \%$ (see Figure A2 and Table A2). According to the Wilcoxon signed ranks test, the relative frequencies of zero loss avoidance levels are significantly different between the SNG treatment and the PGG treatment $(p=.002)$. The one-tailed test between SNG and IND is also significant $(p=.000)$ the distribution on the intermediate level is not significantly different to the SNG. To interpret this evidence, we hypothesize that the behavior of the SNG is guided by its two components the group decision and the individual decision. ${ }^{12}$ Subjects react to the outcomes of the collective goods game and also react to the outcomes of the game of chance. To show the effect of the outcomes of these components in the data we examine the dynamics of the experiment once more.

Observation 5: Individual loss avoidance levels of the SNG treatment are negatively correlated to the lagged losses of the other group members (conditional cooperation effect), and positively correlated to an own lagged loss (social responsibility effect).

Table 4 reports fixed effects regression results on the determinants of the individual loss avoidance levels. For the regressions we consider the following independent variables. In row $\mathrm{f}$, we report the effect of the own loss incurred in the previous period, LaggedLoss. Row $\mathrm{g}$ records the effect of the lagged average loss incurred by the other group members, Lagged Others'Loss. Row $\mathrm{h}$ refers to the individual's lagged loss avoidance levels, LaggedLoss

12. In contrast to the PGG where subjects contribute to the public good a certain amount, in the SNG they contribute a lottery whose odds are determined by their chosen loss avoidance level. In this way we speak of the two components of the SNG. 
TABLE 4

Dynamics of the Loss Effect: Fixed Effects Regression of Individual Loss Avoidance Levels

\begin{tabular}{lcc}
\hline Independent Variables & (8) PGG & (9) IND \\
\hline (a) $S N G \times$ LaggedLoss & $0.0250^{* *}(0.0114)$ & $-0.0379^{*}(0.0201)$ \\
(b) $S N G \times$ LaggedOthers'Loss & $0.0120(0.00862)$ & - \\
(c) $S N G \times$ LaggedLossAvoidanceLevel & $0.0974^{* *}(0.0454)$ & $-0.0943(0.0779)$ \\
(d) $S N G \times$ Period & $-0.0783(0.254)$ & $-0.895^{* * *}(0.216)$ \\
(e) SNG & $-2.210(2.736)$ & $1.621(3.682)$ \\
(f) LaggedLoss & - & $0.0609^{* * *}(0.0175)$ \\
(g) LaggedOthers'Loss & $-0.0310^{* * *}(0.00832)$ & - \\
(h) LaggedLossAvoidanceLevel & $0.334^{* * *}(0.0342)$ & $0.644^{* * *}(0.0716)$ \\
(i) Period & $-0.702^{* * *}(0.206)$ & $0.103(0.174)$ \\
(j) Constant & $22.99^{* * *}(2.105)$ & $14.00^{* * *}(3.332)$ \\
Observations & 1,512 & 1,512 \\
Independent groups & 112 & 112 \\
$R^{2}$ & 0.238 & 0.455 \\
\hline
\end{tabular}

Notes: Dependent variable: average loss avoidance level of subject $i$ in period $t$, Independent variables: $S N G$ (Dummy 1 if SNG, 0 if PGG and IND, respectively), LaggedLoss (incurred loss in $t-1$ ), LaggedOthers'Loss (average of incurred loss of others in $t-1$ ), LaggedLossAvoidanceLevel (subject's chosen loss avoidance level in $t-1$ ), Period (current period $1,2, \ldots, 10)$ and interaction variables $S N G \times Y$ where $Y=\{$ LaggedLoss, LaggedLossAvoidanceLevel, LaggedOthers'Loss, Period $\}$. Standard errors in parentheses.

${ }^{* * *} p<.01,{ }^{* *} p<.05,{ }^{*} p<.1$.

AvoidanceLevel. These variables are interacted with the variable $S N G$ (see rows a: $S N G \times$ LaggedLoss, b: SNG $\times$ LaggedOthers'Loss, and c: $S N G \times$ LaggedLossAvoidanceLevel). The results for the SNG treatment are reported jointly with the PGG treatment in column 8 and with the IND treatment in column 9. From row $g$ of column 8 we find that the loss avoidance levels depend negatively on the lagged losses of the others. This conditional cooperation effect (see Neugebauer et al. 2009 and references therein) appears to be similar in both treatments since the coefficient of the interacted variable indicates no significant differences between the SNG and the PGG treatments (row b). The social responsibility effect in the SNG treatment is evidenced from the significance of the interacted variable in row a. ${ }^{13}$ If subjects experience a loss, they tend to increase their loss avoidance levels in the next period. Note that the reaction to an own loss is even more significant in the IND treatment, see rows a and f of column 9. For the IND treatment this effect is in line with ex post rational adjustment behavior (Selten and Buchta 1994; Selten and Stöcker 1986). In hindsight, subjects

13. Note that the lagged loss avoidance level corresponds to the lagged own loss in the PGG treatment, but in the SNG treatment this equivalence is not given, owed to the stochastic component. Therefore, we check the effect of the lagged loss through the lines (a), (c), and (h) of column 8. Line (h) indicates a path dependence effect; the loss avoidance level positively correlates with the loss avoidance level in the previous period. in the IND treatment could have earned a higher payoff in the previous period. In adaptive manner, subjects increase rather than decrease their loss avoidance level if they incurred a loss in the prior period, and they rather decrease than increase their loss avoidance level when the loss did not occur. Owed to the limited liability of subjects in the SNG, however, this behavior cannot be explained by ex post rational adjustments. Thus, we may want to refer to this reaction as a social responsibility effect. Subjects act as if they anticipated regret for their losses and the efficiency loss they impose on the group, and they avoid further losses by increasing their loss avoidance levels. Similar to the PGG, however, the actions are more frequently chosen in the interior than on the boundaries of the action space. This fact could be a source for why our results differ to those observed in the related binary choice task by Berger and Hershey (1994). Berger and Hershey suggested that risk rather decreases the cooperation levels in PGGs whereas, in our study, risk improves cooperation levels.

\section{SUMMARY AND CONCLUSIONS}

This article introduces the safety net game in which members of a group choose avoidance levels to prevent a likely individual loss which is socially indemnified within the group. The safety net game involves the moral hazard problem since the group members have limited 
liability for any loss they impose on the group and since their individual actions are unobservable by the others. If a loss occurs, it is hard to prove, in hindsight, that it occurred due to the lack of loss avoidance measures or rather due to chance. We discussed the safety net game theoretically and reported experimental results and compared it to two control treatments; the fullliability individual decision task and the nonrisky linear public goods game. For the safety net game and for the public goods game, standard theory predicts free riding. Under full liability, in contrast, theory predicts the choice of the maximal loss avoidance level. As for the full liability control treatment, the experimental data support the theoretical prediction that the loss avoidance levels are lower in the safety net game than under full liability. Although this result is not surprising, the difference between the two treatments is surprising as it is far smaller than predicted. There are two reasons for this deviation from the theoretical prediction. On one hand, the full liability treatment elicited relatively high levels of risk taking as only $88 \%$ of the disposable resources were employed on loss avoidance rather than the predicted $100 \%$. On the other hand, the safety net game treatment elicited lower risk taking levels as $56 \%$ of the resources were employed rather than the predicted $0 \%$. Thus, despite no differences in the theoretical prediction, the observed avoidance levels of the safety net game were significantly higher than the $42 \%$ average loss avoidance level observed in the public goods game. However, the dynamics of the two games were statistically indistinguishable from another as subjects adjust their loss avoidance levels conditionally on the others' observed losses.

Our observations suggest that the higher loss avoidance levels compared to the public goods game result from the risk exposure of cooperative individuals. To show cooperation under hidden action the individual loss avoidance level must be sufficiently high to weather the move of nature. So instead of hiding behind the outcomes of nature as suggested in the moral hazard line of reasoning, subjects' cooperation levels are shifted from the lower half of action space to the upper half in an attempt to make a loss less likely to occur. If a subject fails to avoid the loss in one period, our data show that the loss avoidance level is increased in response to this loss in the following period. We interpret this effect as socially responsible behavior. Subjects behave as if responsible for imposing a loss on the group even though they have only limited liability. However, we also observe in line with the theory that some subjects ride free on the others, the predicted zero loss avoidance level is chosen in $18 \%$ of all decisions. The number of free-riders increases with repetition, however, following a similar pattern as in the public goods game. The loss avoidance levels evolve inversely to the observed losses incurred by the other group members. So, risk taking increases gradually as response to the observed erosion of moral standards.

This observation is consistent with the findings of Grossman (1992); in his empirical study on the effect of federal deposit insurance on thrift risk taking he writes (p. 819): "While newly insured thrifts undertook less risk than their uninsured counterparts, moral hazard emerged gradually. The results [...] indicate that after having been insured for approximately five years an insured institution's risk taking surpassed that of its uninsured counterpart [...]. Once insured, however, thrift managers felt free to undertake more risk."

Although the moral hazard problem observed in the data is not as dramatic as expected in theory, the increase of risk taking suggests a gradual erosion of social responsibility. There are at least two political measures that can be taken to mitigate this erosion which have also been discussed in the literature (Holmstrom 1979). First, the insurance literature suggests that the inclusion of a deductible upon a realized loss can enhance moral standards as participants' liability is increased. The crucial question in this context, indeed, is on the size of the deductible that would lead to the desirable outcome. Second, the literature on agency problems suggests a monitoring of the agent's actions. Here the question arises how probable a monitoring must be owed to the costs of monitoring in safety nets. A sensible policy might be the introduction of monitoring in the frame of an alert system. Upon a realized loss and increasingly with every further loss, monitoring efforts must be increased. Monitoring must be accompanied by a reasonable sanction of defectors who-in the worst case - might face exclusion from the safety net. Such measures do not only discipline defectors, but also serve as a general moral support for social responsibility. These relevant questions on sensible political measures to support the maintenance of moral standards, which can also be addressed within the safety net game experiment, obviously call for further research. 


\section{APPENDIX}

TABLE A1

Random Effects Regression: Absence of Order Effect in the SNG

\begin{tabular}{lccccc}
\hline $\begin{array}{l}\text { Independent } \\
\text { Variables }\end{array}$ & $\begin{array}{c}\text { SNG_PGG vs. } \\
\text { SNG_IND }\end{array}$ & $\begin{array}{c}\text { SNG1 vs. } \\
\text { SNG2_PGG }\end{array}$ & $\begin{array}{c}\text { SNG1 vs. } \\
\text { SNG2_IND }\end{array}$ & $\begin{array}{c}\text { SNG2_PGG vs. } \\
\text { SNG2_IND }\end{array}$ & $\begin{array}{c}\text { SNG1_PGG vs. } \\
\text { SNG1_IND }\end{array}$ \\
\hline $\begin{array}{l}\text { Run2Dummy } \\
\text { Sun2Dummy }\end{array}$ & $5.377(3.829)$ & $3.733(4.907)$ & $7.893(5.024)$ & $4.160(4.390)$ & $6.595(6.325)$ \\
Period & $-0.338(0.245)$ & $-0.0496(0.287)$ & $-0.449(0.298)$ & $-0.399(0.371)$ & $-0.277(0.321)$ \\
Constant & $-1.289^{* * *}(0.173)$ & $-1.334^{* * *}(0.166)$ & $-1.334^{* * *}(0.172)$ & $-1.383^{* * *}(0.262)$ & $-1.195^{* * *}(0.227)$ \\
Observations & $33.65^{* * *}(2.708)$ & $33.43^{* * *}(2.833)$ & $33.43^{* * *}(2.901)$ & $37.16^{* * *}(3.104)$ & $30.13^{* * *}(4.472)$ \\
Independent groups & 280 & 210 & 210 & 140 & 140 \\
Wald test & 28 & 21 & 21 & 74.25 & 14 \\
\end{tabular}

Notes: SNG1 and SNG2 stand for first and second run of SNG treatment;_PGG and_IND for the PGG and IND sessions, respectively. The dependent variable is the average loss avoidance level of a group in period $t$. Run2Dummy is a binary variable, which takes value 0 for the first and 1 for the second ten periods. Period is the period number of the treatment and takes values $\{1, \ldots, 10\}$ accordingly. Standard errors in parentheses.

${ }^{* * *} p<.01,{ }^{* *} p<.05,{ }^{*} p<.1$.

TABLE A2

Loss Avoidance Levels: Descriptive Statistics

\begin{tabular}{lcccc}
\hline & & IND & SNG & PGG \\
\hline Relative frequency $x=0$ & First period & $0.00 \%$ & $9.82 \%$ & $25.00 \%$ \\
& Last period & $0.00 \%$ & $30.36 \%$ & $57.14 \%$ \\
Relative frequency $x<25$ & Average & $0.36 \%$ & $18.13 \%$ & $35.36 \%$ \\
& First period & $8.93 \%$ & $29.46 \%$ & $48.21 \%$ \\
Relative frequency $25 \leq x<50$ & Last period & $7.14 \%$ & $58.93 \%$ & $75.00 \%$ \\
& Average & $5.54 \%$ & $43.30 \%$ & $59.82 \%$ \\
Relative frequency $x=50$ & First period & $44.64 \%$ & $37.50 \%$ & $19.64 \%$ \\
& Last period & $37.50 \%$ & $30.36 \%$ & $17.64 \%$ \\
Average absolute level & Average & $43.21 \%$ & $35.89 \%$ & $18.39 \%$ \\
& First period & $46.43 \%$ & $33.04 \%$ & $32.14 \%$ \\
$N$ & Last period & $55.36 \%$ & $10.71 \%$ & $7.14 \%$ \\
& Average & $51.25 \%$ & $20.80 \%$ & $21.79 \%$ \\
& First period & 43.09 & 34.68 & 26.23 \\
& Last period & 44.61 & 20.75 & 12.38 \\
& Average & 44.08 & 28.31 & 20.94
\end{tabular}

TABLE A3

OLS Regression for Various Segments of the Loss Avoidance Level Distribution

\begin{tabular}{lcrrr}
\hline Model & Variable & SNG & PGG & IND \\
\hline 2-step OLS & Intercept & 0.370 & 0.200 & 0.002 \\
& Loss avoidance level & 0.008 & 0.005 & 0.001 \\
& D25 & 0.032 & 0.085 & -0.031 \\
& D25 $\times$ Loss avoidance level & 0.001 & 0.011 & 0.016 \\
OLS & Adj $R^{2}$ & .991 & .994 & -976 \\
& Intercept & 0.358 & 0.110 & 0.090 \\
& Loss avoidance level & 0.009 & 0.013 & .009 \\
& Adj $R^{2}$ & .987 & .936 & .793 \\
\hline
\end{tabular}

Notes: The dependent variable is the cumulative relative frequency of the loss avoidance level. D25 is a binary variable, which takes value 0 for loss avoidance level below 25 and 1 otherwise.

\footnotetext{
Standard errors in parentheses.
}

${ }^{* * *} p<.01,{ }^{* *} p<.05,{ }^{*} p<.1$. 
FIGURE A1

Cumulative Distribution of Loss Avoidance Levels by Periods in SNG

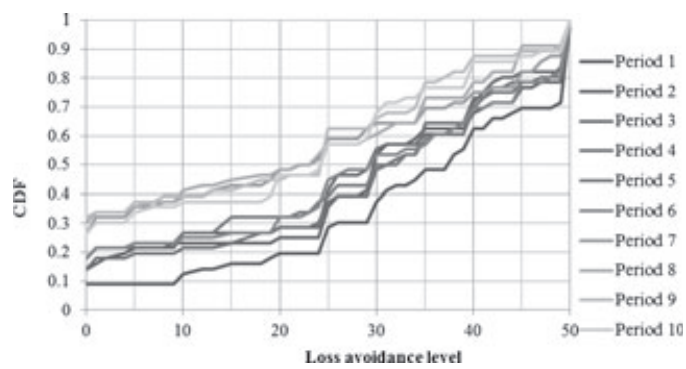

FIGURE A2

Cumulative Distribution of Loss Avoidance Levels by Periods in PGG

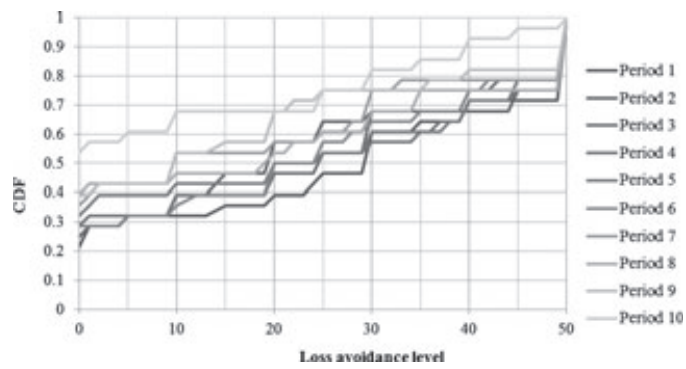

FIGURE A3

Cumulative Distribution of Loss Avoidance Levels by Periods in IND

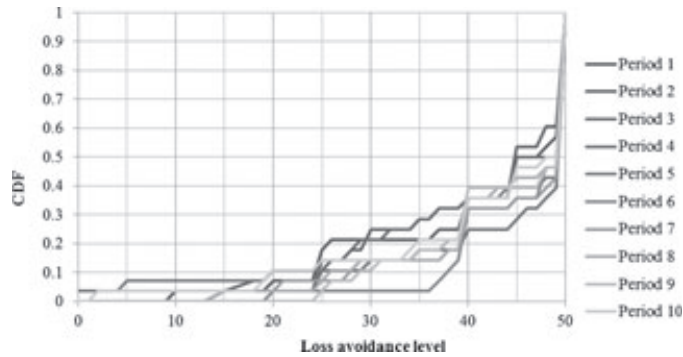

INSTRUCTIONS (TRANSLATION)

(Any treatment) Welcome to the experiment! Please switch off your mobile telephone! Please pay attention to the following instructions and inform the instructor in case you experience problems in understanding. During the entire experiment you are not allowed to communicate with any other participant. If you do communicate with other participants you will have to abandon the experiment immediately and you lose all claims regarding payment. By participating in the experiment you contribute to the experimental research on individual decision making. Therefore, it is important that you make all your decisions on your own!

Instructions independent of the order

\section{The IND Treatment}

In the experiment you are going to make individual decisions in ten periods.

In every period, you will receive an endowment of 150 Eurocent. You decide on the allocation of an integer amount between 0 and 50 Eurocents in order to avoid a personal loss of 100 Eurocents.

With every allocated Eurocent, you reduce the likelihood of the loss. The incurrence of the loss is determined by chance and depends on your allocated amount.

In each period the computer randomly determines a personal threshold level, an integer between 1 and 50. If your allocated amount is greater or equal to this threshold level the loss is avoided. If the allocated amount is smaller you incur a loss of 100 Eurocents.

Note: The threshold level always exceeds 0 , and never exceeds 50. Hence, the loss is certain if your allocated amount is 0 and loss avoidance is certain if your allocated amount is 50 Eurocents. Hence, your payoff in any period equals

\section{0 (your endowment)}

- your allocated amount to avoid the loss

- 100 (if you incur the loss)

You make all your decisions on the computer. After each period the following information is displayed in a table: period, allocated amount, threshold level, your incurred loss, period payoff.

\section{The SNG Treatment}

In the experiment you are going to interact with three other participants in a group of four for ten periods. The composition of the group will stay the same throughout the entire experiment. The identity of the group members will be randomly determined at the beginning and will not be revealed to you at any time.

In every period, you will receive an endowment of 150 Eurocent. You decide on the allocation of an integer amount between 0 and 50 Eurocents in order to avoid a personal loss of 100 Eurocents.

With every allocated Eurocent, you reduce the likelihood of the loss. The incurrence of the loss is determined by chance and depends on your allocated amount.

In each period the computer randomly determines a personal threshold level, an integer between 1 and 50 .

If your allocated amount is greater or equal to this threshold level the loss is avoided. If the allocated amount is smaller you incur a loss of 100 Eurocents.

Note: The threshold level always exceeds 0 , and never exceeds 50. Hence, the loss is certain if your allocated amount is 0 and loss avoidance is certain if your allocated amount is 50 Eurocents.

If you incur a loss it is equally divided between the members of your group. In other words, you share your loss with the others. Likewise, you share the incurred losses of the other group members. Hence, your payoff in any period equals

\section{0 (your endowment)}

- your allocated amount to avoid the loss 
- 100 (if you incur the loss)/4

- 100 (for each loss incurred by the other three group members $) / 4$

[In other words, 150 - your allocated amount - incurred losses of the group/4]

You make all your decisions on the computer. After each period the following information is displayed in a table: period, allocated amount, threshold level, your incurred loss, sum of all incurred losses, your quarter of all incurred losses, period payoff.

\section{The PGG Treatment}

In the experiment you are going to interact with three other participants in a group of four for ten periods. The composition of the group will stay the same throughout the entire experiment. The identity of the group members will be randomly determined at the beginning and will not be revealed to you at any time.

In every period, you will receive an endowment of 150 Eurocent. You decide on the allocation of an integer amount between 0 and 50 Eurocents in order to avoid a personal loss of 100 Eurocents.

With every allocated Eurocent, you reduce the maximum loss by 2 Eurocents.

If you incur a loss it is equally divided between the members of your group. In other words, you share your loss with the others. Likewise, you share the incurred losses of the other group members. Hence, your payoff in any period equals

\section{0 (your endowment)}

- your allocated amount to avoid the loss

- [100 (loss) - 2 times your allocated amount $] / 4$

- $[100$ (loss) - 2 times allocated amount (for each of the other three group members)]/4

[In other words, 150 - your allocated amount - incurred losses of the group/4]

You make all your decisions on the computer. After each period the following information is displayed in a table: period, allocated amount, your incurred loss, sum of all incurred losses, your quarter of all incurred losses, period payoff.

\section{Computer Supported Quiz (Description)}

Before starting the SNG and the PGG treatment subjects faced a comprehension test. The test involved two screens. On the first screen, subjects were prompted to insert eight (four in PGG) integers between 1 and 50 (without being told that that was part of a quiz). On the second screen, subjects faced these numbers in a different order, representing allocated amounts to avoid the loss by four fictitious players and their threshold levels (not in PGG). On the basis of these numbers and the random arrangement of the numbers on the screen, subjects had to compute the payoff for each fictitious player. Subjects confirmed their computations by a press on a button. However, only if they had passed the test could they continue the experiment. The treatment started when all subjects had passed.

\section{REFERENCES}

Abbring, J., P. Chiappori, and J. Pinquet. "Moral Hazard and Dynamic Insurance Data." Journal of the European Economic Association, 1, 2003, 767-820.
Anderhub, V., M. Königstein, and S. Gächter. "Efficient Contracting and Fair Sharing in a Principal-Agent Experiment." Experimental Economics, 5, 2002, 5-25.

Berg, J. E., L. A. Daley, J. W. Dickhaut, and J. O'Brien. "Moral Hazard and Risk Sharing: Experimental Evidence." Research in Experimental Economics, 5, 1992, $1-34$.

Berger, L. A., and J. C. Hershey. "Moral Hazard, Risk Seeking, and Free Riding." Journal of Risk and Uncertainty, 9(2), 1994, 173-86.

Breyer, F., P. Zweifel, and M. Kifmann. Gesundheitsökonomik. Berlin, Heidelberg, New York: Springer, 1994.

Croson, R., E. Fatas, and T. Neugebauer. "Reciprocity, Matching and Conditional Cooperation in Two Public Goods Games." Economics Letters, 87, 2005, 95-102.

Dickinson, D. "The Voluntary Contributions Mechanism with Uncertain Group Payoffs." Journal of Economic Behavior \& Organization, 35(4), 1998, 517-33.

Di Mauro, C. "Ex Ante and Ex Post Moral Hazard in Compensation for Income Losses: Results from an Experiment." Journal of Socio-Economics, 31, 2002, 253-71.

Dionne, G., M. Maurice, J. Pinquet, and C. Vanasse. "The Role of Memory in Long-Term Contracting with Moral Hazard: Empirical Evidence in Automobile Insurance." Manuscript, HEC Montreal, 2005.

Epstein, S. "Testing Principal-Agent Theory, Experimental Evidence." Research in Experimental Economics, 5, 1992, 35-60.

Fehr, E., S. Gächter, and G. Kirchsteiger. "Reciprocity as a Contract Enforcement Device: Experimental Evidence." Econometrica, 65, 1997, 833-60.

Fischbacher, U. "z-Tree: Zurich Toolbox for Ready-made Economic Experiments." Experimental Economics, 10, 2007, 171-8.

Gächter, S., and M. Königstein. "Design a Contract! A Simple Principal-Agent Problem as a Classroom Experiment." Journal of Economic Education, 40(2), 2009, $173-87$.

Gangadharan, L., and V. Nemes. "Experimental Analysis of Risk and Uncertainty in Provisioning Private and Public Goods.” Economic Inquiry, 47(1), 2009, 146-64.

Greiner, B. "An Online Recruitment System for Economic Experiments. Forschung und wissenschaftliches Rechnen." GWDG Bericht 63. Ges. für Wiss. Datenverarbeitung, 2004, 79-93.

Grossman, R. "Deposit Insurance, Regulation, and Moral Hazard in the Thrift Industry: Evidence from the 1930's." American Economic Review, 82, 1992, 800-21.

Güth, W., W. Klose, M. Königstein, and J. Schwalbach. "An Experimental Study of a Dynamic Principal-Agent Relationship." Managerial and Decision Economics, 27, 1998, 327-41.

Hasson, R., A. Löfgren, and M. Visser. "Climate Change in a Public Goods Game: Investment Decision in Mitigation Versus Adaptation." Ecological Economics, 70(2), 2010, 331-8.

Hey, J. D. Experiments in Economics. Oxford, UK: Blackwell, 1991.

Holmstrom, B. "Moral Hazard and Observability." Bell Journal of Economics, 10, 1979, 74-91.

Isaac, R. M., J. M. Walker, and S. H. Thomas. "Divergent Evidence on Free Riding: An Experimental Examination of Possible Explanations." Public Choice, 43, 1984, 113-49.

Kahneman, D., and A. Tversky. "The Framing of Decisions and the Psychology of Choice." Science, 185, 1981, $1124-31$. 
Keser, C., and M. Willinger. "Principals' Principles When Agents' Actions Are Hidden." International Journal of Industrial Organization, 18, 2000, 163-85.

. "Theories of Behavior in Principal-Agent Relationships with Hidden Action." European Economic Review, 51, 2007, 1514-33.

Ledyard, J. "Public Goods: A Survey of Experimental Research," in Handbook of Experimental Economics, edited by J. Kagel and A. Roth. Princeton, NJ: Princeton University Press, 1995, 111-94.

Lohse, T., J. R. Robledo, and U. Schmidt. "Self-Insurance and Self-Protection as Public Goods." Journal of Risk and Insurance, 79(1), 2012, 57-76.

Neugebauer, T., J. Perote, U. Schmidt, and M. Loos. "Selfish-Biased Conditional Cooperation: On the Decline of Contributions in Repeated Public Goods Experiments." Journal of Economic Psychology, 30, 2009, 52-60.

Pallage, S., and C. Zimmermann. "Voting on Unemployment Insurance Generosity." International Economic Review, 42(4), 2001, 903-24.
Pauly, M. V. "The Economics of Moral Hazard: A Comment." American Economic Review, 58, 1968, 531-7.

Selten, R., and J. Buchta. "Experimental Sealed-Bid First Price Auctions with Directly Observed Bid Functions," in Games and Human Behavior: Essays in the Honor of Amnon Rapoport's 60th Birthday, edited by D. Budescu, I. Erev, and R. Zwick. Mahwah, NJ: Lawrence Erlbaum Associates, 1994, 79-104.

Selten, R., and R. Stöcker. "End Behavior in Sequences of Finite Prisoners' Dilemma Supergames: A Learning Theory Approach." Journal of Economic Behavior \& Organization, 7, 1986, 47-70.

Shanley, J., and P. J. Grossman. "Paradise to Parking Lots: Creation Versus Maintenance of a Public Good." Journal of Socio-Economics, 36(4), 2007, 523-36.

Wolfe, J., and J. Goddeeris. "Adverse Selection, Moral Hazard, and Wealth Effects in the Medigap Insurance Market." Journal of Health Economics, 10, 1991, 433-59. 